\title{
Primary Lateral Sclerosis
}

National Cancer Institute

\section{Source}

National Cancer Institute. Primary Lateral Sclerosis. NCI Thesaurus. Code C129933.

A progressive neurodegenerative disorder affecting upper motor neurons, characterized by progressive muscle weakness. 